\title{
Tau-Path Following Method for Solving the Riccati Equation with Fractional Order
}

\author{
Muhammed I. Syam, ${ }^{1}$ Hani I. Siyyam, ${ }^{2}$ and Ibrahim Al-Subaihi ${ }^{2}$ \\ ${ }^{1}$ Department of Mathematical Sciences, United Arab Emirates University, Al Ain 15551, United Arab Emirates \\ ${ }^{2}$ Department of Mathematics, Taibah University, Al Madinah, Saudi Arabia \\ Correspondence should be addressed to Muhammed I. Syam; m.syam@uaeu.ac.ae
}

Received 15 December 2013; Accepted 30 January 2014; Published 5 March 2014

Academic Editor: Marta B. Rosales

Copyright (C) 2014 Muhammed I. Syam et al. This is an open access article distributed under the Creative Commons Attribution License, which permits unrestricted use, distribution, and reproduction in any medium, provided the original work is properly cited.

A formulation for the fractional Legendre functions is constructed to find the solution of the fractional Riccati equation. The fractional derivative is described in the Caputo sense. The method is based on the Tau Legendre and path following methods. Theoretical and numerical results are presented. Analysis for the presented method is given.

\section{Introduction}

Recently, many papers on fractional boundary value problems have been studied extensively. Several forms of them have been proposed in standard models, and there has been significant interest in developing numerical schemes for their solutions. Several numerical techniques are used to solve such problems such as Laplace and Fourier transforms [1,2], Adomian decomposition and variational iteration methods $[3,4]$, eigenvector expansion [5], differential transform and finite differences methods [6,7], power series method [8], collocation method [9], and wavelet method [10, 11]. Many applications of fractional calculus on various branches of science such as engineering, physics, and economics can be found in $[12,13]$. Considerable attention has been given to the theory of fractional ordinary differential equations and integral equations $[14,15]$. Additionally, the existence of solutions of ordinary and fractional boundary value problems using monotone iterative sequences has been investigated by several authors [16-20].

We consider the Riccati equation with fractional orders of the form

$$
\begin{array}{r}
a(x) D^{\alpha} y(x)+b(x) y(x)+c(x) y^{2}(x)=g(x), \\
x \in(0,1), 0<\alpha<1,
\end{array}
$$

$$
y(0)=y_{\mathrm{ini}},
$$

where $a, b, c$, and $g$ are continuous functions on $[0,1]$ and $y_{\mathrm{ini}}$ is a constant.

Riccati equation with fractional order has been discussed by many researchers using different techniques such as collocation method based on Muntz polynomials [21], homotopy perturbation method [22], and series solution method [23].

In this paper we study the Tau-path following method for solving the Riccati equation with fractional order. We organize this paper as follows. In Section 2, we present basic definitions and results of fractional derivatives. We extend basic results to path following method for the fractional case. In Section 3, we introduce the fractional-order Legendre Tau method with path following method for solving the Riccati equation with fractional order. In Section 4, we present some numerical results to illustrate the efficiency of the presented method. Finally, we conclude with some comments in the last section.

\section{Preliminaries}

In this section, we review the definition and some preliminary results of the Caputo fractional derivatives, as well as, the definition of the fractional-order Legendre functions and their properties. 
Definition 1. The Riemann-Liouville fractional integral operator $I^{\alpha}$ of order $\alpha>0$ on the usual Lebesgue space $L_{1}[0,1]$ is given by

$$
\begin{gathered}
I^{\alpha} f(x)=\frac{1}{\Gamma(\alpha)} \int_{0}^{x} \frac{f(t)}{(x-t)^{1-\alpha}} d t, \\
I^{0} f(x)=f(x),
\end{gathered}
$$

where $\Gamma(\zeta)=\int_{0}^{\infty} t^{\zeta-1} e^{-t} d t$ is the Euler Gamma function, see $[12,24]$.

For any $f \in L_{1}[0,1], \alpha, \beta \geqslant 0$, and $\gamma>-1$, the following properties hold:

(1) $I^{\alpha}$ exists for any $x \in[0,1]$,

(2) $I^{\alpha} I^{\beta}=I^{\alpha+\beta}$,

(3) $I^{\alpha} I^{\beta}=I^{\beta} I^{\alpha}$,

(4) $I^{\alpha} x^{\gamma}=(\Gamma(\gamma+1) / \Gamma(\alpha+\gamma+1)) x^{\alpha+\gamma}$.

Definition 2. The Caputo fractional derivative of order $\alpha$ is defined by

$$
D^{\alpha} f(x)=I^{n-\alpha} D^{n} f(x)=\frac{1}{\Gamma(n-\alpha)} \int_{0}^{x} \frac{f^{(n)}(t)}{(x-t)^{\alpha-n+1}} d t
$$

provided that the integral exists, where $n=[\alpha]+1$ and $[\alpha]$ is the integer part of the positive real number $\alpha, x>0$.

The Caputo fractional derivative satisfies the following properties for $f \in L_{1}[0,1]$ and $\alpha, \beta \geqslant 0$ :

(1) $D^{\alpha} I^{\alpha} f(x)=f(x)$,

(2) $I^{\alpha} D^{\alpha} f(x)=f(x)-\sum_{k=0}^{n-1} f^{(k)}\left(0^{+}\right)\left(x^{k} / k !\right)$,

(3) $D^{\alpha} D^{\beta} f(x)=D^{\beta} D^{\alpha} f(x)=D^{\alpha+\beta} f(x), x>0$,

(4) $D^{\alpha} c=0$, where $c$ is constant,

(5) $D^{\alpha} x^{\gamma}=\left\{\begin{array}{cc}0, & \gamma<\alpha, \gamma \in\{0,1,2, \ldots\} \\ (\Gamma(\gamma+1) / \Gamma(\gamma-\alpha+1)) x^{\gamma-\alpha}, & \text { otherwise }\end{array}\right\}$,

(6) $D^{\alpha}\left(\sum_{k=0}^{m} c_{i} f_{i}(x)\right)=\sum_{k=0}^{m} c_{i} D^{\alpha} f_{i}(x)$, where $c_{1}, c_{2}, \ldots$, $c_{m}$ are constants.

The basic concept of this paper is the Legendre polynomials. For this reason, we study some of their properties.

Definition 3. The Legendre polynomials $\left\{\mathscr{L}_{k}(x): k=\right.$ $0,1,2, \ldots\}$ are the eigenfunctions of the Sturm-Liouville problem

$$
\left(\left(1-x^{2}\right) \mathscr{L}_{k}^{\prime}(x)\right)^{\prime}+k(k+1) \mathscr{L}_{k}(x)=0, \quad x \in[-1,1] .
$$

Among the properties of the Legendre polynomials we list the following properties:

(1) $\int_{-1}^{1} \mathscr{L}_{i}(x) \mathscr{L} j(x) d x=(2 /(2 i+1)) \delta_{i j}$, where $\delta_{i j}=$
$\left\{\begin{array}{l}0, i \neq j \\ 1, i=j\end{array}\right\}$,
(2) $\mathscr{L}_{i+1}(x)=((2 i+1) /(i+1)) x \mathscr{L}_{i}(x)-(i /(i+1)) \mathscr{L}_{i-1}(x)$, for $i \geqslant 1$,
(3) $\mathscr{L}_{i}( \pm 1)=( \pm 1)^{i}$.

In order to use these polynomials on the interval $[0,1]$, we define the shifted Legendre polynomials by $S_{i}(z)=\mathscr{L}_{i}(2 z-$ $1)$. Using the change of variable $x=2 z-1, S_{i}(z)$ has the following properties:

(1) $\int_{0}^{1} S_{i}(z) \operatorname{Sj}(z) d z=(1 /(2 i+1)) \delta_{i j}$, (2) $S_{i+1}(z)=((2 i+1) /(i+1))(2 z-1) S_{i}(z)-(i /(i+1)) S_{i-1}(z)$,
for $i \geqslant 1$,

(3) $S_{i}(0)=(-1)^{i}$ and $S_{i}(1)=1$.

The analytic closed form of the shifted Legendre polynomials of degree $i$ is given by

$$
S_{i}(z)=\sum_{k=0}^{i}(-1)^{i+k} \frac{(i+k) !}{(i-k) !} \frac{z^{k}}{(k !)^{2}} .
$$

One of the common and efficient methods for solving fractional differential equations of order $\alpha>0$ is using the series expansion of the form $\sum_{k=0}^{i} c_{k} t^{\alpha k}$. For this reason, we define the fractional-order Legendre function by $F_{i}^{\alpha}(t)=$ $S_{i}\left(t^{\alpha}\right)$. Using the properties of the shifted Legendre polynomials and the change of variable $z=t^{\alpha}$, it is easy to show that

(1) $\left(\left(t-t^{1+\alpha}\right) F_{i}^{\alpha^{\prime}}(t)\right)^{\prime}+i(i+1) \alpha^{2} t^{\alpha-1} F_{i}^{\alpha}(t)=0, t \in(0,1)$,

(2) $F_{i}^{\alpha}(t)=((2 i+1) /(i+1))\left(2 t^{\alpha}-1\right) F_{i}^{\alpha}(t)-(i /(i+1)) F_{i-1}^{\alpha}(t)$, for $i \geqslant 1$,

(3) $F_{0}^{\alpha}(t)=1$ and $F_{1}^{\alpha}(t)=2 t^{\alpha}-1$,

(4) $F_{i}^{\alpha}(0)=(-1)^{i}$ and $F_{i}^{\alpha}(1)=1$.

In addition, $\left\{F_{i}^{\alpha}(t): i=0,1,2, \ldots\right\}$ are orthogonal functions with respect to the weight function $w(t)=t^{\alpha-1}$ on $(0,1)$ with

$$
\int_{0}^{1} F_{i}^{\alpha}(t) F_{j}^{\alpha}(t) w(t) d t=\frac{1}{(2 i+1) \alpha} \delta_{i j} .
$$

The closed form of $F_{i}^{\alpha}(t)$ is given by

$$
F_{i}^{\alpha}(t)=\sum_{k=0}^{i}(-1)^{i+k} \frac{(i+k) !}{(i-k) !} \frac{t^{\alpha k}}{(k !)^{2}} .
$$

Using properties (4) and (5) of the Caputo fractional derivative, one can see that

$$
\begin{aligned}
D^{\alpha} F_{i}^{\alpha}(t)=\sum_{k=1}^{i}(-1)^{i+k} \frac{(i+k) !}{(i-k) !(k !)^{2}} \\
\quad \times \frac{\Gamma(k \alpha+1)}{\Gamma((k-1) \alpha+1)} t^{(k-1) \alpha} .
\end{aligned}
$$

In the next theorem, we state one of the main properties of the fractional Legendre functions which will be used later in this paper. 
Theorem 4. For any nonnegative integers $p$ and $q$,

$$
F_{p}^{\alpha}(t) F_{q}^{\alpha}(t)=\sum_{k=0}^{[(p+q) / 2]} A_{2 k} F_{p+q-2 k}^{\alpha}(t),
$$

where

$$
\begin{aligned}
A_{2 k}= & (2 p+2 q-4 k+1)(2 k) !(2 p-2 k) ! \\
& \left.\times(2 q-2 k) ! 2^{p+q-k}((p+q-k) !)^{2}\right) \\
& \times\left(2^{p+q-k}(2 p+2 q-2 k+1)(k !)^{2}\right. \\
& \left.\quad \times((p-q) !)^{2}((q-k) !)^{2}(2 p+2 q-2 k) !\right)^{-1} .
\end{aligned}
$$

Proof. For any nonnegative integers $p$ and $q$,

$$
\mathscr{L}_{p}(x) \mathscr{L}_{q}(x)=\sum_{k=0}^{[(p+q) / 2]} A_{2 k}^{\alpha} \mathscr{L}_{p+q-2 k}(x),
$$

where

$$
\begin{aligned}
A_{2 k}= & (2 p+2 q-4 k+1)(2 k) !(2 p-2 k) ! \\
& \left.\times(2 q-2 k) ! 2^{p+q-k}((p+q-k) !)^{2}\right) \\
\times & \left(2^{p+q-k}(2 p+2 q-2 k+1)(k !)^{2}\right. \\
& \left.\quad \times((p-q) !)^{2}((q-k) !)^{2}(2 p+2 q-2 k) !\right)^{-1} .
\end{aligned}
$$

For the proof of this case, see [25]. Using the change of variables $x=2 z-1$ and $z=t^{\alpha}$, we obtain the result of the theorem.

Another important result which will facilitate applying the Tau method for fractional case is given in Theorem 5.

Theorem 5. Let $u \in C[0,1]$ and $u^{\prime}(t)$ be a piecewise continuous function on $[0,1]$. Then, $u(t)$ can be written in the infinite expansion as $u(t)=\sum_{k=0}^{\infty} u_{k} F_{k}^{\alpha}(t)$, where

$$
u_{k}=(2 i+1) \alpha \int_{0}^{1} u(t) F_{k}^{\alpha}(t) w(t) d t,
$$

and $w(t)=t^{\alpha}$.

Proof. If $u \in C^{1}[-1,1]$ and $u^{\prime \prime}(x)$ is a piecewise continuous function on $[-1,1], \sum_{k=0}^{\infty} v_{k} \mathscr{L}_{k}(x)$ converges uniformly to $u(x)$ on $[-1,1]$, see $[26-28]$. Let $h:[0,1] \rightarrow[-1,1]$ by

$$
h(t)=2 t^{\alpha}-1
$$

Since $h(t)$ is a bijective continuous function, $\sum_{k=0}^{\infty} u_{k} F_{k}^{\alpha}(t)$ converges uniformly to $u(t)$ on $[0,1]$. The value of $u_{k}$ follows from the orthogonality relation of $\left\{F_{i}^{\alpha}(t): i=0,1,2, \ldots\right\}$ with respect to the weight function $w(t)=t^{\alpha-1}$ on $[0,1]$

Theorem 6 gives the relation between the coefficient of the series solution of $D^{\alpha} u(t)$ and the coefficients of the series expansion of $u(t)$.

Theorem 6. Let $u \in C^{1}[0,1]$ and $u^{\prime \prime}(t)$ be a piecewise continuous function on $[0,1]$. Then, $\sum_{k=0}^{\infty} u_{k}^{(\alpha)} F_{k}^{\alpha}(t)$ converges uniformly on $[0,1]$ to $D^{\alpha} u(t)$, where

$$
\begin{aligned}
& u_{k}^{(\alpha)}=\sum_{j=k+1}^{\infty} a_{j k} u_{j}, \\
& a_{j k}=(2 k+1) \alpha \int_{0}^{1} D^{\alpha} F_{j}^{\alpha}(t) F_{k}^{\alpha}(t) w(t) d t \\
& \quad \text { for } k=0,1,2, \ldots, j=k+1, k+2, \ldots .
\end{aligned}
$$

Proof. Let $S_{n}(t)=\sum_{k=0}^{n} u_{k} F_{k}^{\alpha}(t)$ for $n=0,1,2, \ldots$. From Theorem $5, S_{n}(t)$ converges uniformly to $u(t)$ on $[0,1]$. Since $u \in C^{1}[0,1]$ and $u^{\prime \prime}(t)$ is a piecewise continuous function on $[0,1]$,

$$
\frac{d}{d t}\left(\operatorname{Lim}_{n \rightarrow \infty} S_{n}(t)\right)=\operatorname{Lim}_{n \rightarrow \infty}\left(\frac{d}{d t} S_{n}(t)\right)
$$

and $(d / d t) S_{n}(t)$ converges uniformly to $(d / d t) u(t)$ on $[0,1]$. Thus, $\int_{0}^{x}\left(S_{n}^{\prime}(t) /(x-t)^{\alpha}\right) d t$ converges uniformly to $\int_{0}^{x}\left(u^{\prime}(t) /(x-t)^{\alpha}\right) d t$ on $[0,1]$ which gives the result of the theorem. The value of $a_{j k}$ follows from the orthogonality relation of $\left\{F_{i}^{\alpha}(t): i=0,1,2, \ldots\right\}$ with respect to the weight function $w(t)=t^{\alpha-1}$ on $[0,1]$.

\section{Fractional-Order Legendre Tau-Path Following Method}

In this section, we present a numerical method for solving problem (1)-(2). We use the fractional-order Legendre Tau method to discretize problem (1)-(2). The result is a nonlinear system. The initial guess that is used in the standard methods for solving the produced nonlinear system is one of the challenges. To overcome this problem, we use the path following method. Approximate the solution $y(x), a(x), b(x)$, $c(x)$, and $g(x)$ in terms of the fractional-order Legendre functions as follows:

$$
\begin{gathered}
Y_{N}(x)=\sum_{k=0}^{N+1} y_{k} F_{k}^{\alpha}(x), \quad a_{N}(x)=\sum_{k=0}^{N+1} a_{k} F_{k}^{\alpha}(x), \\
b_{N}(x)=\sum_{k=0}^{N+1} b_{k} F_{k}^{\alpha}(x), \quad c_{N}(x)=\sum_{k=0}^{N+1} c_{k} F_{k}^{\alpha}(x), \\
g_{N}(x)=\sum_{k=0}^{N+1} g_{k} F_{k}^{\alpha}(x) .
\end{gathered}
$$

Thus, $D^{\alpha} y(x)$ can be approximated by

$$
D^{\alpha} Y_{N}(x)=\sum_{k=0}^{N} y_{k}^{(\alpha)} F_{k}^{\alpha}(x),
$$


where $y_{k}^{(\alpha)}$ is given by Theorem 6. For $Y_{N}$, the residual is given by

$$
\begin{aligned}
R\left(Y_{N}\right)= & a_{N}(x) D^{\alpha} Y_{N}(x)+b_{N}(x) Y_{N}(x)+c_{N}(x) Y_{N}^{2}(x) \\
& -g_{N}(x)
\end{aligned}
$$

which can be written as

$$
\begin{aligned}
R\left(Y_{N}\right)= & \left(\sum_{k=0}^{N+1} a_{k} F_{k}^{\alpha}(x)\right)\left(\sum_{k=0}^{N} y_{k}^{(\alpha)} F_{k}^{\alpha}(x)\right) \\
& +\left(\sum_{k=0}^{N+1} b_{k} F_{k}^{\alpha}(x)\right)\left(\sum_{k=0}^{N+1} y_{k} F_{k}^{\alpha}(x)\right) \\
& +\left(\sum_{k=0}^{N+1} c_{k} F_{k}^{\alpha}(x)\right)\left(\sum_{k=0}^{N+1} y_{k} F_{k}^{\alpha}(x)\right)^{2}-\sum_{k=0}^{N+1} g_{k} F_{k}^{\alpha}(x)
\end{aligned}
$$

or

$$
\begin{aligned}
R\left(Y_{N}\right)= & \sum_{k=0}^{2 N+1} \sum_{j=0}^{k} a_{j} y_{k-j}^{(\alpha)} F_{j}^{\alpha}(x) F_{k-j}^{\alpha}(x) \\
& +\sum_{k=0}^{2 N+2} \sum_{j=0}^{k} b_{j} y_{k-j} F_{j}^{\alpha}(x) F_{k-j}^{\alpha}(x) \\
& +\sum_{k=0}^{3 N+3} \sum_{i=0}^{k} \sum_{j=0}^{k-i} c_{i} y_{j} y_{k-i-j} F_{j}^{\alpha}(x) F_{k-i-j}^{\alpha}(x) F_{i}^{\alpha}(x) \\
& -\sum_{k=0}^{N+1} g_{k} F_{k}^{\alpha}(x),
\end{aligned}
$$

where $a_{j}=0, b_{j}=0, y_{j}=0, c_{j}=0$ for $j \geq N+2$, and $y_{j}^{(\alpha)}=0$ for $j \geq N+1$. Using Theorem 4 , we can rewrite the residual as

$$
\begin{aligned}
R & \left(Y_{N}\right) \\
= & \sum_{k=0}^{2 N+1} \sum_{j=0}^{k} \sum_{l=0}^{[k / 2]} A_{l} a_{j} y_{k-j}^{(\alpha)} F_{k-2 l}^{\alpha}(x) \\
& +\sum_{k=0}^{2 N+2} \sum_{j=0}^{k} \sum_{l=0}^{[k / 2]} A_{l} b_{j} y_{k-j} F_{k-2 l}^{\alpha}(x) \\
& +\sum_{k=0}^{3 N+3} \sum_{i=0}^{k} \sum_{j=0}^{k-i[(k-i) / 2][(k-2 l) / 2]} \sum_{l=0} \sum_{m=0} A_{m} A_{l} c_{i} y_{j} y_{k-i-j} F_{k-2 l-2 m}^{\alpha}(x) \\
& -\sum_{k=0}^{N+1} g_{k} F_{k}^{\alpha}(x) .
\end{aligned}
$$

Since we are interested in the first $n$ terms only, we ignore higher order terms and we rewrite the residual as

$$
R\left(Y_{N}\right)=\sum_{k=0}^{N} d_{k} F_{k}^{\alpha}(x)
$$

Orthogonalize the residual with respect to the fractionalorder Legendre function as follows

$$
\int_{0}^{1} R\left(Y_{N}\right) F_{j}^{\alpha}(x) w(x) d x=0, \quad \text { for } j=0: N,
$$

where $w(x)=x^{\alpha-1}$. Therefore, (25) leads to the elementwise equation

$$
d_{j}=\frac{1}{(2 k+1) \alpha}, \quad \text { for } j=0: N \text {. }
$$

Therefore, we can write (26) as a system of $N$ nonlinear equations in $(N+1)$ unknowns as

$$
\Omega(Y)=R_{1},
$$

where $Y=\left[\begin{array}{llll}y_{0} & y_{1} & \cdots & y_{N+1}\end{array}\right]^{T}, R_{1}=(1 / \alpha)\left[\begin{array}{llll}1 & 1 / 3 & \cdots\end{array}\right.$ $1 /(2 N+1)]^{T}$, and $T$ means the transpose of the matrix.

From the initial condition (2), one can see that

$$
y_{\text {ini }}=Y_{N}(0)=\sum_{k=0}^{N+1}(-1)^{k} y_{k}
$$

or

$$
y_{\text {ini }}=\Lambda Y \text {, }
$$

where $\Lambda=\left[\begin{array}{llll}1 & -1 & \cdots & (-1)^{N+1}\end{array}\right]^{T}$. From systems (27) and (29), we obtain a system of $(N+1)$ nonlinear equations in $(N+1)$ unknowns

$$
\Psi(Y)=R,
$$

where $\Psi: \mathfrak{R}^{N+1} \rightarrow \mathfrak{R}^{N+1}$ by $\Psi(Y)=\left[\begin{array}{c}\Omega(Y) \\ \Lambda\end{array}\right]$ and $R=$ $\left[\begin{array}{c}R_{1} \\ y_{\text {ini }}\end{array}\right] \in \mathfrak{R}^{N+1}$.

The standard methods for solving system (30), such as secant method and Newton method, need a good initial guess which is not available. To overcome this problem, we look for another method which does not depend on the initial guess. The promise technique is the path following method which is described as follows. From (23), we can see that the function $\Omega$ is the sum of three terms. Two of them are linear systems which are $\sum_{k=0}^{2 N+1} \sum_{j=0}^{k} \sum_{l=0}^{[k / 2]} A_{l} a_{j} y_{k-j}^{(\alpha)} F_{k-2 l}^{\alpha}(x)$ and $\sum_{k=0}^{2 N+2} \sum_{j=0}^{k} \sum_{l=0}^{[k / 2]} A_{l} b_{j} y_{k-j} F_{k-2 l}^{\alpha}(x)$. The third term is nonlinear system which is

$$
\sum_{k=0}^{3 N+3} \sum_{i=0}^{k} \sum_{j=0}^{k-i[(k-i) / 2][(k-2 l) / 2]} \sum_{l=0} A_{m=0} A_{l} c_{i} y_{j} y_{k-i-j} F_{k-2 l-2 m}^{\alpha}(x) .
$$

Thus, we can rewrite $\Omega$ as

$$
\Omega(Y)=A_{1} Y+A_{2} Y+\Omega_{1}(Y),
$$

where $A_{1}$ and $A_{2}$ are two $N \times(N+1)$ matrices and $\Omega_{1}$ : $\mathfrak{R}^{N+1} \rightarrow \mathfrak{R}^{N}$ is a nonlinear function of $Y$. Hence, system (30) can be written as

$$
\Psi(Y)=A Y+\Psi_{1}(Y)=R,
$$


where $A=\left[\begin{array}{c}A_{1} \\ \Lambda\end{array}\right]$ is $(N+1) \times(N+1)$ matrix and $\Psi_{1}: \mathfrak{R}^{N+1} \rightarrow$ $\mathfrak{R}^{N+1}$ is a nonlinear function of $Y$ given by $\Psi_{1}=\left[\begin{array}{c}A_{2}+\Omega_{1}(Y) \\ \mathbf{0}_{1 \times(N+1)}\end{array}\right]$. Define the function $H: \mathfrak{R}^{N+1} \times[0,1] \rightarrow \mathfrak{R}^{N+1}$ by

$$
\begin{aligned}
H(Y, \lambda) & =\lambda(\Psi(Y)-R)+(1-\lambda) A Y \\
& =A Y+\lambda\left(\Psi_{1}(Y)-R\right) .
\end{aligned}
$$

Thus, $H$ satisfies the following properties

(1) $H \in C^{\infty}\left(\mathfrak{R}^{N+1} \times[0,1]\right)$,

(2) $H(\mathbf{0}, 0)=\mathbf{0}$,

(3) $(\partial H / \partial Y)(\mathbf{0}, 0)=A$.

It is worth to mention that $A$ is a nonsingular matrix since $A Y=R$ is the system produced by the following problem:

$$
\begin{gathered}
a(x) D^{\alpha} y(x)=g(x), \quad x \in(0,1), 0<\alpha<1, \\
y(0)=y_{\text {ini }},
\end{gathered}
$$

which has a unique solution. Hence, it follows from the implicit function theorem that there is a smooth curve $\gamma$ : $I \rightarrow \mathfrak{R}^{N+1}$ for some open interval $I$ containing zero such that $\gamma(0)=0, \gamma^{\prime}(0) \neq 0, \operatorname{rank}\left(H^{\prime}(\gamma(s))\right)=N+1$, and

$$
H(\gamma(s))=0
$$

for all $s \in I$. Consider the solution $\gamma(s)=(Y(s), \lambda(s))$ (parametrized for convenience with respect to arc length) such that $\gamma(0)=(\mathbf{0}, 0)$. The solution curve $H^{-1}(Y(0), \lambda(0))$ should be either diffeomorphic to the circle or to the real line. Since the solution point $(\mathbf{0}, 0)$ is unique for $\lambda=0$, it follows that $\gamma$ cannot be closed, and hence, it is diffeomorphic to the real line. Since $\gamma$ is a smooth curve, $Y(s)$ is bounded for $\lambda(s) \in[0,1]$. Moreover, the curve $\gamma$ reaches the level $\lambda=1$ after a finite arc length $s_{1}$. This means, $\gamma\left(s_{1}\right)=(\bar{Y}, 1)$, and hence, $\bar{Y}$ is the zero of $\Psi(Y)-R$. Thus, we can take $I=[0,1]$. For more details about the proof, [29-31]. To apply path following method numerically, we differentiate (36) to get

$$
H^{\prime}(\gamma(0)) \gamma^{\prime}(0)=0 \text {, }
$$

which implies that $\gamma^{\prime}(0)$ is orthogonal to all rows of $H^{\prime}(\gamma(0))$. Thus, for all $s \in[0,1]$,

(1) $\operatorname{det}\left(\begin{array}{c}H^{\prime}(\gamma(s)) \\ \gamma^{\prime}(s)^{T}\end{array}\right)>0$,

(2) $\left\|\gamma^{\prime}(s)\right\|=1$, where $\|\cdot\|$ is the Euclidean norm,

(3) $H^{\prime}(\gamma(s)) \gamma^{\prime}(s)=0$.

We use the predictor-corrector method to numerically trace the curve $\gamma$. The predictor step is the Euler-predictor which is given by

$$
X=Z+h t\left(H^{\prime}(Z)\right)
$$

TABLE 1

\begin{tabular}{lc}
\hline$\alpha$ & Error \\
\hline 0.5 & $1 * 10^{-9}$ \\
0.9 & $2 * 10^{-10}$ \\
0.99 & $1.2 * 10^{-10}$ \\
0.9999 & $1.7 * 10^{-12}$ \\
\hline
\end{tabular}

where $Z$ is a point along the curve $\gamma$, and $h>0$ is a fixed step size. The corrector step is the Gauss-Newton corrector which is given by

$$
W=X-H^{\prime}(X)^{+} H^{\prime}(X),
$$

where $H^{\prime}(X)^{+}=\left(H^{\prime}(X)\right)^{T}\left(H^{\prime}(X) H^{\prime}(X)^{T}\right)^{-1}$. For more details, see [31]. Thus, we will start from $Z_{0}=(\mathbf{0}, 0)$ and then generate the sequence $Z_{1}, Z_{2}, Z_{3}, \ldots$ We stop our procedure at $Z_{k}$ when the last component of $Z_{k} \leq 1$ and the last component of $Z_{k+1}>1$. We can write $Z_{k}$ as $Z_{k}=\left(Y_{k}, \lambda_{k}\right)$. Thus, $Y_{k}$ will be the approximate solution to the system (33).

\section{Numerical Results}

In this section, we implement the Tau-Path following method to the nonlinear fractional Riccati differential equations. Two examples of nonlinear fractional Riccati differential equations are solved to show the efficiency of the presented method.

Example 7. Consider the following initial value problem [32]:

$$
\begin{gathered}
D^{\alpha} y(x)-2 y(x)+y^{2}(x)=1, \quad x \in(0,1), 0<\alpha<1, \\
y(0)=0 .
\end{gathered}
$$

The matrices in (33) are

$$
\begin{gathered}
A=\left[\begin{array}{ccccc}
1 & 0 & 0 & \cdots & 0 \\
0 & 1 & 0 & \cdots & 0 \\
\vdots & \ddots & \ddots & \ddots & \vdots \\
0 & \cdots & 0 & 1 & 0 \\
1 & -1 & \cdots & (-1)^{N} & (-1)^{N+1}
\end{array}\right], \\
A_{2}=\left[\begin{array}{cccc}
-2 & 0 & 0 & \cdots \\
0 & -2 & 0 & \cdots \\
\vdots & \ddots & \ddots & \ddots \\
0 & \cdots & 0 & -2
\end{array}\right], \quad R=\left[\begin{array}{c}
1 \\
0 \\
\vdots \\
0 \\
0
\end{array}\right] .
\end{gathered}
$$

It is easy to see that $|\operatorname{det}(A)|=1$ which means $A$ is nonsingular. Define the error by

$$
e(x, \alpha)=D^{\alpha} Y_{N}(x)-2 Y_{N}(x)+Y_{N}^{2}(x)-1 .
$$

Then, the absolute maximum of the error for different values of $\alpha$ are given in Table 1 for $N=16$.

The exact solution when $\alpha=1$ is $y(x)=1+$ $\sqrt{2} \tanh (\sqrt{2} x+(1 / 2) \log ((\sqrt{2}-1) /(\sqrt{2}+1)))$. The graphs of 
TABle 2

\begin{tabular}{lc}
\hline$\alpha$ & Error \\
\hline 0.5 & $2.7 * 10^{-10}$ \\
0.9 & $8.6 * 10^{-11}$ \\
0.99 & $5.4 * 10^{-11}$ \\
0.9999 & $2.1 * 10^{-13}$ \\
\hline
\end{tabular}

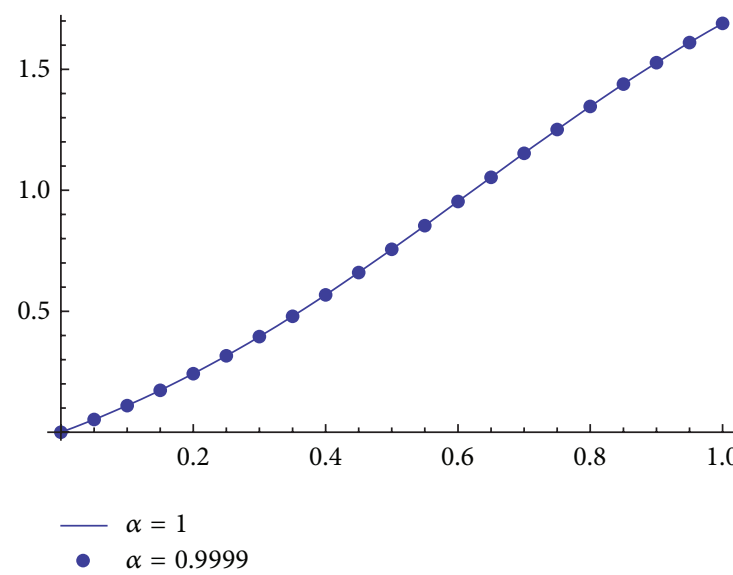

FIGURE 1: The graphs of the approximate solution when $\alpha=0.9999$ and the graph of the exact solution when $\alpha=1$.

the approximate solution when $\alpha=0.9999$ and the graph of the exact solution when $\alpha=1$ are given in Figure 1 .

Example 8. Consider the following initial value problem [32]:

$$
\begin{gathered}
D^{\alpha} y(x)-y^{2}(x)=t^{2}, \quad x \in(0,1), 0<\alpha<1, \\
y(0)=1 .
\end{gathered}
$$

The matrices in (33) are

$$
\begin{gathered}
A=\left[\begin{array}{ccccc}
1 & 0 & 0 & \cdots & 0 \\
0 & 1 & 0 & \cdots & 0 \\
\vdots & \ddots & \ddots & \ddots & \vdots \\
0 & \cdots & 0 & 1 & 0 \\
1 & -1 & \cdots & (-1)^{N} & (-1)^{N+1}
\end{array}\right], \\
A_{2}=\left[\begin{array}{cccc}
0 & 0 & 0 & \cdots \\
0 & 0 & 0 & \cdots \\
\vdots & \ddots & \ddots & \ddots \\
0 & \cdots & 0 & 0
\end{array}\right]
\end{gathered}
$$

It is easy to see that $|\operatorname{det}(A)|=1$ which means $A$ is nonsingular. Define the error by

$$
e(x, \alpha)=D^{\alpha} Y_{N}(x)-Y_{N}^{2}(x)-x^{2} .
$$

Then, the absolute maximum of the error for different values of $\alpha$ is given in Table 2 for $N=16$.

The exact solution when $\alpha=1$ is $y(x)=$ $\left(x\left(J_{-3 / 4}\left(x^{2} / 2\right) \Gamma(1 / 4)+J_{3 / 4}\left(x^{2} / 2\right) \Gamma(3 / 4)\right)\right) /\left(J_{1 / 4}\left(x^{2} / 2\right) \Gamma(1 / 4)-\right.$ $\left.2 J_{-1 / 4}\left(x^{2} / 2\right) \Gamma(3 / 4)\right)$ where $J_{\nu}(x)$ is the Bessel function

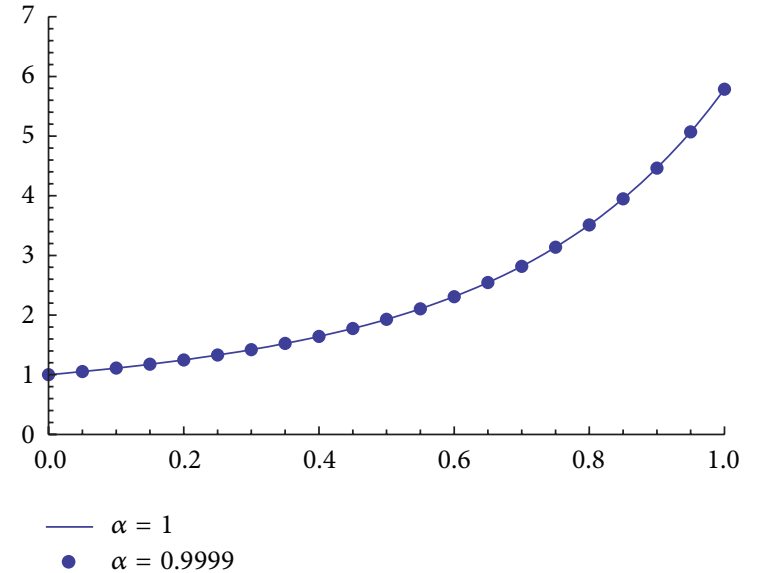

FIGURE 2: The graphs of the approximate solution when $\alpha=0.9999$ and the exact solution when $\alpha=1$.

of first kind. The graphs of the approximate solution when $\alpha=0.9999$ and the exact solution when $\alpha=1$ are given in Figure 2.

\section{Conflict of Interests}

The authors declare that there is no conflict of interests regarding the publication of this paper.

\section{Acknowledgments}

The authors would like to express their appreciation for the valuable comments of the reviewers. The authors also would like to express their sincere appreciation to United Arab Emirates University for the financial support of Grant no. COS/IRG-15/14.

\section{References}

[1] L. Gaul, P. Klein, and S. Kemple, "Damping description involving fractional operators," Mechanical Systems and Signal Processing, vol. 5, no. 2, pp. 81-88, 1991.

[2] I. Podlubny, Fractional Differential Equations: An Introduction to Fractional Derivatives, Fractional Differential Equations, to Methods of Their Solution and Some of Their Applications, Academic Press, New York, NY, USA, 1999.

[3] K. Al-Khaled and S. Momani, "An approximate solution for a fractional diffusion-wave equation using the decomposition method," Applied Mathematics and Computation, vol. 165, no. 2, pp. 473-483, 2005.

[4] S. Das, "Analytical solution of a fractional diffusion equation by variational iteration method," Computers and Mathematics with Applications, vol. 57, no. 3, pp. 483-487, 2009.

[5] L. E. Suarez and A. Shokooh, "An eigenvector expansion method for the solution of motion containing fractional derivatives," Journal of Applied Mechanics, Transactions ASME, vol. 64, no. 3, pp. 629-634, 1997.

[6] A. Arikoglu and I. Ozkol, "Solution of fractional integrodifferential equations by using fractional differential transform 
method," Chaos, Solitons and Fractals, vol. 40, no. 2, pp. 521-529, 2009.

[7] M. M. Meerschaert and C. Tadjeran, "Finite difference approximations for two-sided space-fractional partial differential equations," Applied Numerical Mathematics, vol. 56, no. 1, pp. 80-90, 2006.

[8] Z. M. Odibat and N. T. Shawagfeh, "Generalized Taylor's formula," Applied Mathematics and Computation, vol. 186, no. 1, pp. 286-293, 2007.

[9] Q. M. Al-Mdallal, M. I. Syam, and M. N. Anwar, "A collocationshooting method for solving fractional boundary value problems," Communications in Nonlinear Science and Numerical Simulation, vol. 15, no. 12, pp. 3814-3822, 2010.

[10] Y. LI, "Solving a nonlinear fractional differential equation using Chebyshev wavelets," Communications in Nonlinear Science and Numerical Simulation, vol. 15, no. 9, pp. 2284-2292, 2010.

[11] J. L. Wu, "A wavelet operational method for solving fractional partial differential equations numerically," Applied Mathematics and Computation, vol. 214, no. 1, pp. 31-40, 2009.

[12] A. Kilbas, H. Srivastave, and J. Trujillo, Theory and Applications of Fractional Differential Equations, North-Hollan Mathematics Studies, vol. 204, Elsevier Science, Amsterdam, The Netherlands, 2006.

[13] S. G. Samko, A. A. Kilbas, and O. I. Marichev, Fractional Integrals and Derivative, Theory and Applications, Gordon and Breach, Yverdon, Switzerland, 1993.

[14] V. Lakshmikantham and A. Vatsala, "Basic theory of fractional differential equations," Nonlinear Analysis, Theory, Methods \& Applications, vol. 69, no. 8, pp. 2677-2682, 2008.

[15] S. Zhang, "Existence of solution for a boundary value problem of fractional order," Acta Mathematica Scientia, vol. 26, no. 2, pp. 220-228, 2006.

[16] R. P. Agarwal, M. Benchohra, and S. Hamani, "A survey on existence results for boundary value problems of nonlinear fractional differential equations and inclusions," Acta Applicandae Mathematicae, vol. 109, no. 3, pp. 973-1033, 2010.

[17] M. Al-Refai and M. Ali Hajji, "Monotone iterative sequences for nonlinear boundary value problems of fractional order," Nonlinear Analysis, Theory, Methods and Applications, vol. 74, no. 11, pp. 3531-3539, 2011.

[18] V. Lakshmikantham and A. S. Vatsala, "General uniqueness and monotone iterative technique for fractional differential equations," Applied Mathematics Letters, vol. 21, no. 8, pp. 828834, 2008.

[19] C. V. Pao, Nonlinear Parabolic and Elliptic Equations, Plenum Press, New York, NY, USA, 1992.

[20] K. Oldham and J. Spanier, The Fractional Calculus, Academic, New York, NY, USA, 1974.

[21] S. Esmaeili, M. Shamsi, and Y. Luchko, "Numerical solution of fractional differential equations with a collocation method based on Mntz polynomials," Computers and Mathematics with Applications, vol. 62, no. 3, pp. 918-929, 2011.

[22] N. A. Khan, A. Ara, and M. Jamil, "An efficient approach for solving the Riccati equation with fractional orders," Computers and Mathematics with Applications, vol. 61, no. 9, pp. 2683-2689, 2011.

[23] J. Cang, Y. Tan, H. Xu, and S.-J. Liao, "Series solutions of non-linear Riccati differential equations with fractional order," Chaos, Solitons and Fractals, vol. 40, no. 1, pp. 1-9, 2009.

[24] M. Caputo, "Linear models of dissipation whose Q is almost frequency independent, Part II Geophys," Mathematics \& Physical Sciences, vol. 13, no. 5, pp. 529-539, 1967.
[25] W. A. Al-Salam, "On the product of two Legendre polynomials," Mathematica Scandinavica, vol. 4, pp. 239-242, 1956.

[26] H. I. Siyyam and M. I. Syam, "An accurate solution of the Poisson equation by the Chebyshev-Tau method," Journal of Computational and Applied Mathematics, vol. 85, no. 1, pp. 1-10, 1997.

[27] C. Canuto, M. Y. Hussaini, A. Quarteroni, and T. A. Zang, Spectral Methods in Fluid Dynamics, Springer, New York, NY, USA, 1988.

[28] C. Canuto, M. Y. Hussaini, A. Quarteroni, and T. A. Zang, Spectral Methods: Fundamentals in Single Domains, Springer, New York, NY, USA, 2006.

[29] E. L. Allgower and K. Georg, Numerical Continuation Methods, Springer, New York, NY, USA, 1990.

[30] M. I. Syam and S. M. Al-Sharo, "Collocation-continuation technique for solving nonlinear ordinary boundary value problems," Computers and Mathematics with Applications, vol. 37, no. 10, pp. $11-17,1999$.

[31] H. I. Siyyam and M. I. Syam, "The modified trapezoidal rule for line integrals," Journal of Computational and Applied Mathematics, vol. 84, no. 1, pp. 1-14, 1997.

[32] M. Merdan, "On the solutions fractional Riccati diderential equation with modifed Riemann-Liouville derivative," International Journal of Differential Equations, vol. 2012, pp. 1-17, 2012. 

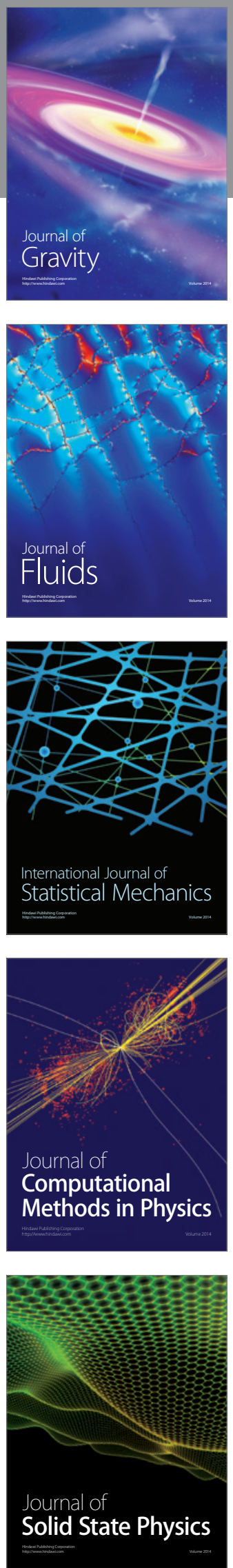

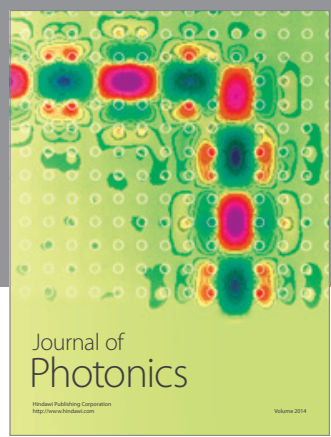

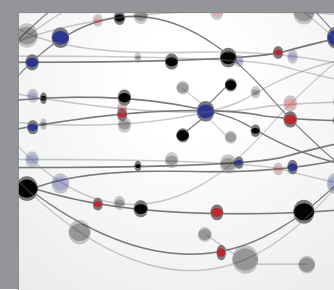

The Scientific World Journal

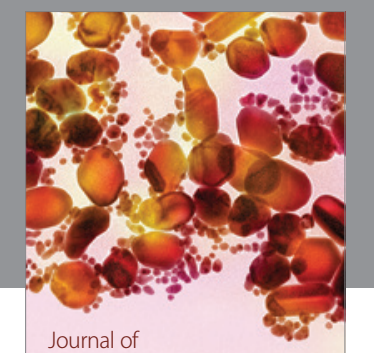

Soft Matter
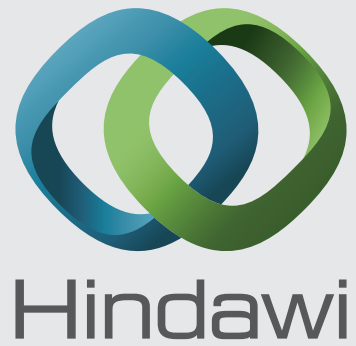

Submit your manuscripts at

http://www.hindawi.com
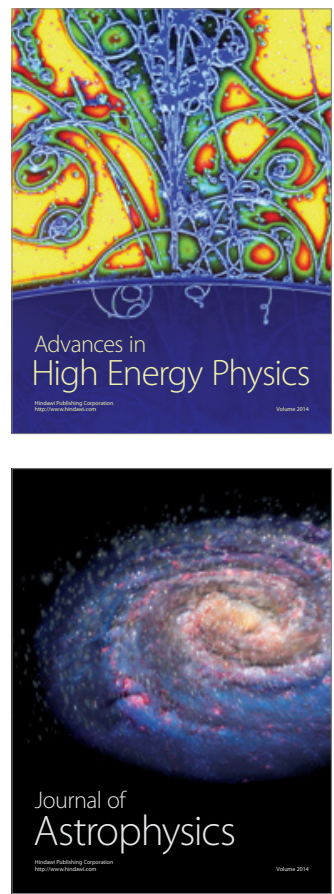
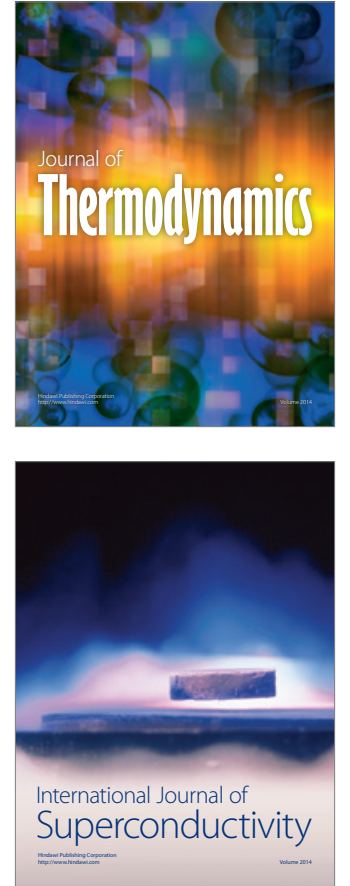
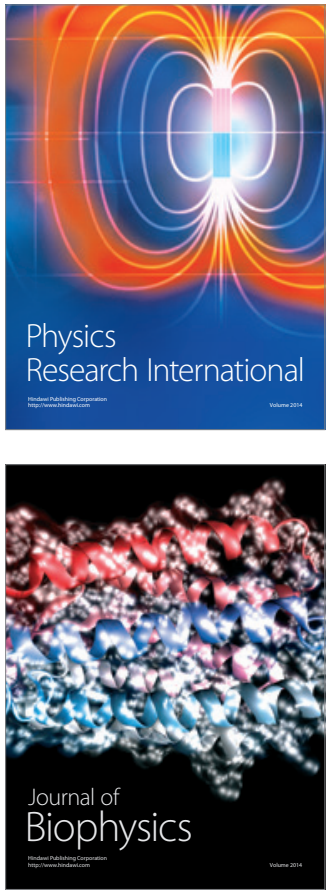
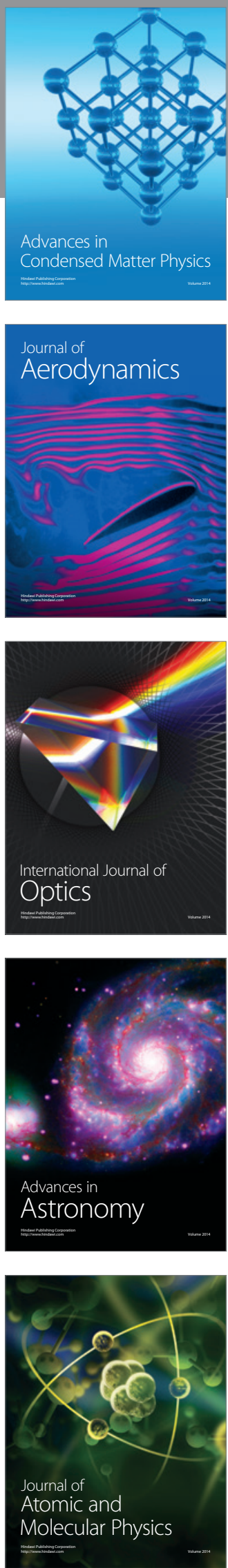